\title{
Magnetotail reconnection: Simulation predictions on magnetic time series
}

\author{
K. Arzner and M. Scholer \\ Centre for Interdisciplinary Plasma Science, Max-Planck-Institut für Extraterrestrische Physik, 85740 Garching, Germany
}

(Received June 5, 2000; Revised October 12, 2000; Accepted November 7, 2000)

\begin{abstract}
In a recent kinetic computer simulation (Arzner and Scholer, 2001), magnetotail reconnection was studied with emphasis on the kinetic (particle) aspect. Complementary, we focus in the present paper on the magnetic fields. Examples of time series of the magnetic field at a fixed position, as predicted for spacecraft probes, are given. Different spatial regions are classified according to their signatures in these time series, and criteria for the proximity to the reconnection point, the post-plasmoid plasma sheet (PPPS) and the boundary layer are derived. The kinetic simulation reveals an instability of the PPPS, which is discussed in terms of an anisotropic 3-layer fluid model with the free energy is provided by the velocity anisotropy $\left(T_{\|}>T_{\perp}\right)$ due to the simultaneous presence of inflowing lobe ions and outflowing ions deflected in the field reversal. In its nonlinear stage, this instability creates higher harmonics and finally leads to turbulence characterized by power law spatial and temporal spectra. The latter allow a direct comparison with GEOTAIL and AMPTE observations. It is found that the simulated and observed turbulence spectra agree well.
\end{abstract}

\section{Introduction}

Spacecraft observations of the magnetic fields during plasma sheet passages have revealed a complex structure with enhanced turbulence during substorms (e.g., Borovsky et al., 1997). On time scales of 10 minutes up to hours, a bistable structure in $B_{x}$ is observed, which is attributed to flapping motions of the tail, exposing the spacecraft alternately to the north- and south half of the plasma sheet. On time scales of $\sim 10$ minutes, well-localized disturbances appear which are connected to bursty bulk flows (Angelopoulos et al., 1992; Hoshino et al., 1994); they may be related to plasmoid passages and caused by elementary reconnection events. On time scales of minutes or smaller, the behavior is found to be turbulent.

In order to understand these observations, both analytical (e.g., Sundaram and Fairfield, 1995; Milovanov et al., 1996; Karimabadi et al., 1999; Kropotkin et al., 1999) and numerical studies have been performed. Among the latter, so-called hybrid simulations (e.g., Krauss-Varban and Omidi, 1995; Kuznetsova et al., 1996; Lin and Swift, 1996) with macroparticle ions and massless electron fluid allow the investigation of collisionless plasmas if electron dynamics can be neglected, i.e. when the electrons are still frozen to the magnetic field but the ions are allowed to decouple. This technique is particularly suitable for large-scale magnetotail simulations since the Earth magnetotail is almost collisionless and the omission of electron inertial scales allows the modeling of substantial parts of the whole tail. In hybrid simulations, the microscopic cause of field line diffusion is considered as a 'black-box' process represented by an anomalous resistivity, or reconnection is modeled by a non-

Copy right (c) The Society of Geomagnetism and Earth, Planetary and Space Sciences (SGEPSS); The Seismological Society of Japan; The Volcanological Society of Japan; The Geodetic Society of Japan; The Japanese Society for Planetary Sciences. diagonal electron pressure tensor or a finite electron mass.

\section{Kinetic Magnetotail Simulations}

The simulation model used by Arzner and Scholer (2001) covers a range of $500 \lambda_{0} \times 125 \lambda_{0}$ with $\lambda_{0}$ the ion inertial length, corresponding to about $100 \mathrm{R}_{\mathrm{E}} \times 25 \mathrm{R}_{\mathrm{E}}$, and it runs over $600 \Omega_{i}^{-1}$ with $\Omega_{i}$ the ion lobe angular gyrofrequency, corresponding to about 10 minutes in real time. The above physical scaling assumes a lobe number density of 0.05 particles per $\mathrm{cm}^{3}$ and a lobe magnetic field strength of $7 \mathrm{nT}$. The coordinate system is righthand cartesian with $+x$ tailward, $+z$ northward and $+y$ dawnward. Distances are measured in units of the ion inertial length at $z=0$, and time is measured in units of $\Omega_{i}^{-1}$ with $\Omega_{i}$ the lobe angular gyrofrequency. In simulation units, the initial Alfvén velocity drops from $v_{A}=1.6$ at the Earthward lobe $(x=0)$ to $v_{A}=0.6$ at the tailward lobe $\left(x=500 \lambda_{0}\right)$. The initial configuration is a tail equilibrium (e.g., Birn et al., 1975; Zwingmann et al., 1990) with a magnetic normal component across the equatorial plane. The initial current sheet density exceeds the lobe density by a factor 2.5 . Reconnection is initiated by a anomalous resistivity at $x_{\eta}=100 \lambda_{0}$ and $z=0$, which is localized within $2 \lambda_{0}$ and decays exponentially in time with a decay time of $250 \Omega_{i}^{-1}$. The boundary conditions for particles and electromagnetic fields are reflecting at the Earthward side and open at the north- and south boundaries. At the north- and south boundaries, a buffer zone of numerical resistivity is applied which damps in- and outgoing waves. Further details of the simulation configuration can be found in Lottermoser and Scholer (1999) and Arzner and Scholer (2001); the numerical code is described in Matthews (1994). The magnetic field is normalized to its lobe value at $x=0$, $\mathbf{B}=( \pm 1,0,0)$. Within the simulation time and for the frequencies considered, any spacecraft motion can be neglected, so that spacecraft time series are approximatively 
time series at fixed positions in the simulation frame of reference.

\section{Instability of the PPPS}

During the simulation, a plasmoid is formed and ejected tailwards with bulk velocity in the order of $v_{A}$. The equatorial region behind the plasmoid contains a new, thin current layer; the so-called post-plasmoid plasma sheet (PPPS) with an enhanced out-of-plane current $j_{y}$. This is illustrated in Fig. 1, showing $j_{y}$ (top) and the bulk velocity $u_{x}$ (middle) at time $t=500 \Omega_{i}^{-1}$, together with an example of the velocity distribution in the PPPS (bottom left) and the bulkand Alfvén velocity profiles at $x=190 \lambda_{0}$ (bottom right). In the course of the simulation, the PPPS becomes unstable, warps and filaments in the wake of the plasmoid (Fig. 1 top). The instability is approximatively convective, i.e. the oscillations are convected tailwards with the reconnection outflow. In the boundary layer of the PPPS and the adjacent lobe, the perturbations are Alfvénic, whereas in the center of the PPPS a simple mode association fails due to the inhomogeneity of the system. A similar instability has also been observed by Karimabadi et al. (1999) in two-dimensional hybrid simulations of reconnection starting from a Harris equilibrium.

\subsection{Linear stage}

The linear stage of the instability was discussed by Arzner and Scholer (2001) in terms of a simple 3-layer fluid model combining the anisotropic velocity distribution $\left(T_{\|}>T_{\perp}\right)$ in the PPPS with the shear flow across the PPPS boundary. In
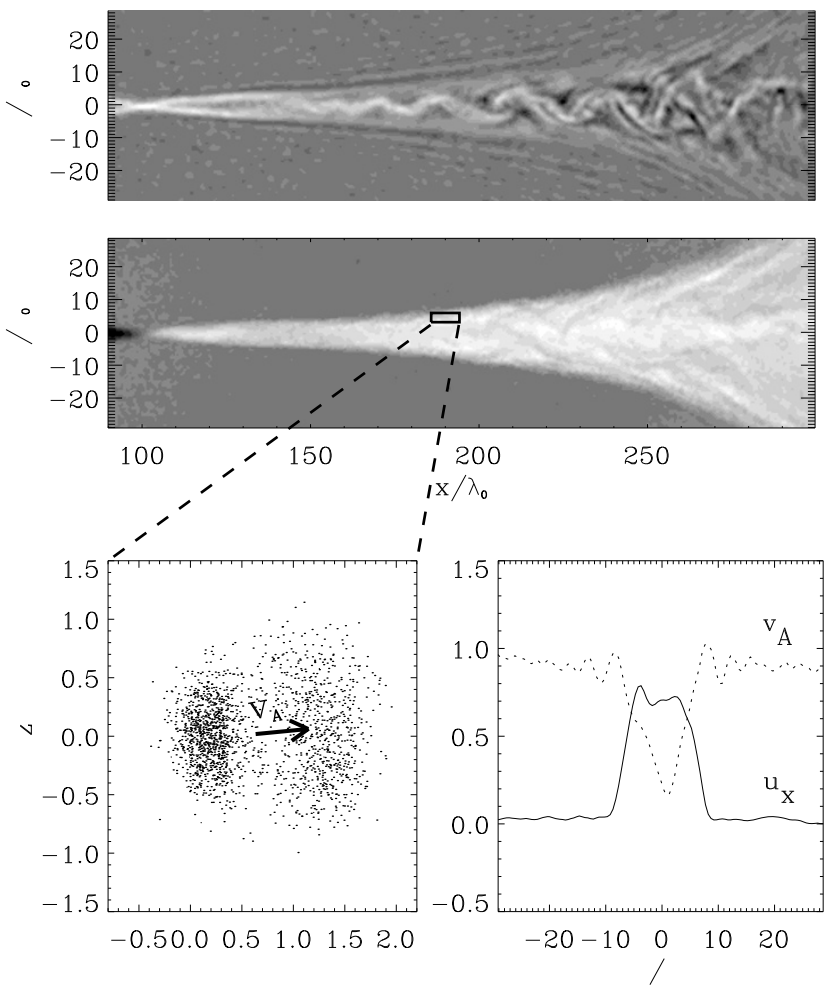

Fig. 1. Top: out-of-plane current $j_{y}$. Middle: bulk velocity $u_{x}$. Bottom: velocity distribution with field-aligned beams (left) and local Alfvén velocity (arrow), bulk- and Alfvén velocity profiles at $x=190 \lambda_{0}$ (right). All graphs represent snapshots at $t=500 \Omega_{i}^{-1}$; the reconnection point is at $x=100 \lambda_{0}$. the following, we briefly repeat the argumentation given by Arzner and Scholer (2001). The velocity anisotropy results from the simultaneous presence of incoming lobe ions and outgoing ions deflected in the field reversal; the responsible electric field is $E_{y} \sim u_{x} B_{z}$ and the process is similar to Speiser-type acceleration. There is an about equal number of in- and outflowing ions with relative velocity $\gtrsim 2 v_{A}^{\text {local }}$ which form a pair of dense, field-aligned beams (Fig. 1 bottom left). From a kinetic point of view, this two-beam configuration drives a nonresonant ion-ion instability (Gary, 1993), whose properties are quite similar to the firehose instability driven by a bi-Maxwellian with equivalent temperature anisotropy. An anisotropic fluid description thus applies. The dispersion relation of the 3-layer fluid model is obtained by requiring that the total pressure and the $z$-displacement are continuous across the PPPS boundaries. The perturbations are assumed to be Alfvénic and propagate along $x$. The dispersion relation can be solved analytically, and yields (simulation frame)

$$
\omega=\frac{V k_{x} \pm k_{x} \sqrt{(\xi+1)\left(\xi \chi_{l} v_{A l}^{2}+\chi_{s} v_{A s}^{2}\right)-\xi V^{2}}}{\xi+1}
$$

where the subscripts $l, s$ refer to lobe and PPPS, $n$ is the density, $V$ is the outflow (PPPS) velocity, $v_{A}$ is the Alfvén velocity, $\xi=\left(n_{l} / n_{s}\right) \sinh k_{x} L /\left(1+\cosh k_{x} L\right)$ is a geometrical parameter depending on the density ratio and the diameter of the PPPS, $2 L$, and $\chi=\left(\beta_{\perp}-\beta_{\|}+2\right) / 2$ is the firehose parameter. In a homogeneous plasma, $\chi=1$ describes (stable) Alfvén waves, and $\chi<0$ indicates firehose instability. In the 3-layer model, instability occurs if

$$
\xi V^{2}>(\xi+1)\left(\xi \chi_{l} v_{A l}^{2}+\chi_{s} v_{A s}^{2}\right) .
$$

From Eq. (2) one recognizes that both the shear flow $(V)$ and the velocity anisotropy $(\chi)$ contribute to instability. For the parameters relevant to the simulation, the free energy is mainly provided by the velocity anisotropy, whereas the bulk velocity shear is insufficient to drive a pure KelvinHelmholtz instability, but plays a auxiliary role. The physical reason for this is that in our two-dimensional simulation the instability propagates along the magnetic field and therefore needs to bend the magnetic field lines, which requires more energy than available in the shear flow. The growth rate $\gamma$ predicted by Eq. (1) has a maximum at $k \lesssim k_{\max }$ and vanishes at $k>k_{\max }$, where $k_{\max }$ depends on $L$ and the density ratio $n_{l} / n_{s}$. The wavelength of maximum growth increases with increasing $L$, in agreement with the simulation (Fig. 3 below). The propagation properties are determined by the modes of the complete 3-layer model, and satisfy $\omega_{r}\left(k_{x}\right) \simeq V k_{x}$ at the wavelength of maximum growth.

The dispersion relation of the anisotropic 3-layer fluid model (Eq. (1)) is shown in Fig. 2 (boldface line), together with the dispersion relation found from the hybrid simulation (contours). Both refer to the simulation frame of reference. The parameters used in the 3-layer model are: $n_{l} / n_{s}=$ $0.57, v_{A l}=0.9 \lambda_{0} \Omega_{i}, v_{A s}=0.4 \lambda_{0} \Omega_{i}, V=0.7 \lambda_{0} \Omega_{i}$, $\chi_{l}=1, \chi_{s}=-1.5, L=4.0 \lambda_{0}$. They correspond to a profile of the PPPS at $x \sim 190 \lambda_{0}$ and $t \sim 500 \Omega_{i}^{-1}$ (cf. Fig. 1 bottom). The dispersion relation of the hybrid simulation is obtained by Fourier analyzing $B_{y}\left(x, z_{0}, t\right)$ at fixed 

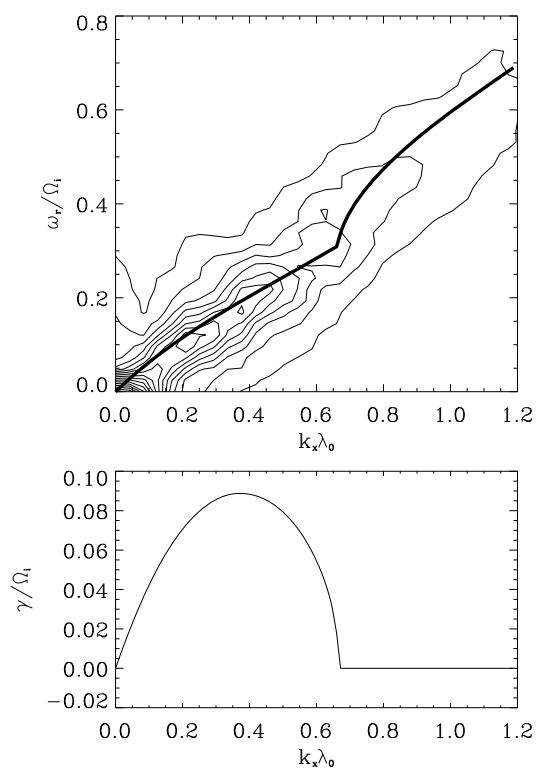

Fig. 2. Top: the dispersion relation (real frequency vs. wave number) of the PPPS instability found in the hybrid simulation (contours), and according to the 3-layer model (boldface line). Bottom: the growth rate of the 3-layer model. The parameters used in Eq. (1) are: $n_{l} / n_{s}=0.57$, $v_{A l}=0.9 \lambda_{0} \Omega_{i}, v_{A s}=0.4 \lambda_{0} \Omega_{i}, V=0.7 \lambda_{0} \Omega_{i}, \chi_{l}=1, \chi_{s}=-1.5$, $L=4.0 \lambda_{0}$.

$z_{0}, 170 \lambda_{0} \leq x \leq 320 \lambda_{0}$, and $240 \Omega_{i}^{-1} \leq t \leq 500 \Omega_{i}^{-1}$. It was verified that the choice of a particular field component and of $z_{0}$ does not affect the dispersion relation, as long as $z_{0}$ is within the region of instability. As can be noticed, there is a good agreement between the 3-layer model prediction and the simulation with regard to the real frequency and the excited wave lengths. The temporal growth rate in the hybrid simulation can be inferred from the spatial growth rate; it is found that $\gamma \sim 0.05$, in qualitative agreement with the 3-layer model prediction $\gamma \sim 0.08$ (Fig. 2 bottom). The difference in the growth rates can be explained by nonlinear saturation.

\subsection{Nonlinear stage}

The instability of the PPPS is particularly distinctly seen in $B_{y}$, hereafter called the Hall field (Fig. 3 top). The presence of a significant Hall field $\left|B_{y}\right| \lesssim 0.4$ is a consequence of ion inertia included in the hybrid simulation and the Alfvén theorem of Hall MHD (e.g., Arzner and Scholer, 2001). As the instability is convective, its temporal evolution can be studied by a sequence of spatial power spectra $S\left(k_{x}\right)$ at cuts along $x$ (Fig. 3 ). In the calculation of the power spectra shown in Fig. 3, a one-dimensional Welch window was applied with half widths marked in Fig. 3. The spectra were averaged over the times $t=490,495$ and $500 \Omega_{i}^{-1}$, during which they do not change significantly. The lefthand plasmoid boundary, which separates particles originating from the lobe and from the initial plasma sheet, is at $x \approx 330 \lambda_{0}$.

In its linear stage and at $x \sim 150 \lambda_{0}$, the spectrum is almost monochromatic with $k_{x} \lambda_{0} \sim 0.3$ (Fig. 3 bottom left) but during propagation, nonlinear interactions create higher harmonics and mixing frequencies (Fig. 3 bottom middle), and lead finally to turbulence in the sense of power law-like

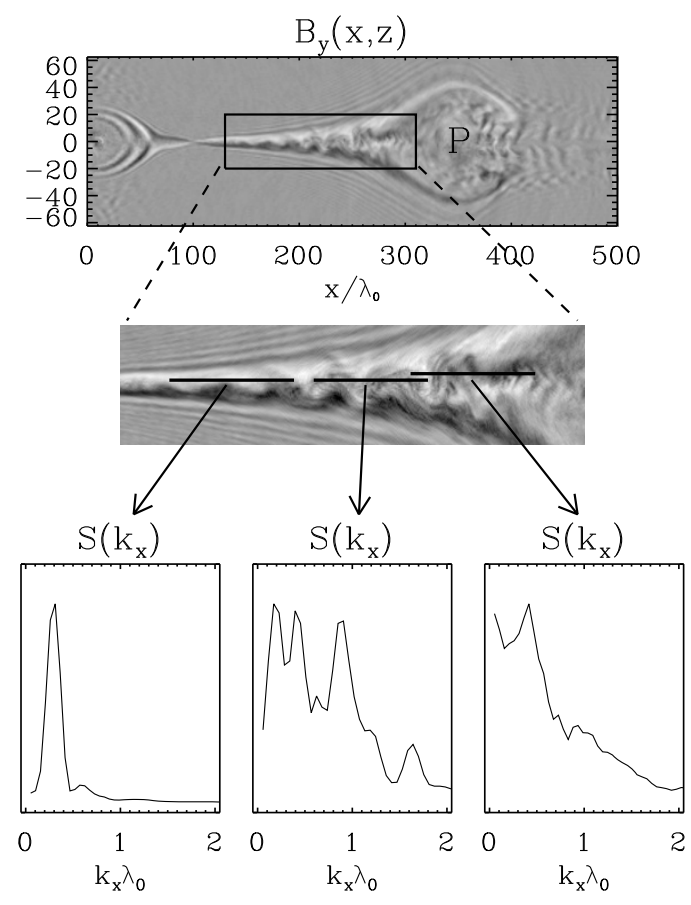

Fig. 3. Top: a snapshot of the Hall magnetic field $B_{y}$ at time $t=500 \Omega_{i}^{-1}$. Center: a close-up of the post-plasmoid plasma sheet showing its instability and turbulence. Bottom: the spatial power spectra (linear scale) along the cuts marked in the center panel. The plasmoid (' $\mathrm{P}$ ') moves to the right.

temporal/spatial wave spectra (Fig. 3 bottom right, Fig. 7 below). Superimposed to this cascading is a drift of the input (fundamental) scale to larger wave lengths, which is associated to the increase of the linearly excited wavelength with increasing PPPS diameter in tailward direction (see above). Nonlinear saturation occurs due to energy transfer to smaller scales. Note that the transition to turbulence follows a classical route of wave cascading. Within a fluid picture, this process can be described as follows. At the boundary of the PPPS, Alfvénic perturbations are excited by the velocity anisotropy due to the field-aligned beams. In the center of the PPPS and in the wake of the plasmoid, the plasma beta is $\gg 1$, so that compressibility must be taken into account. As a result, the Alfvén waves propagating along $x$ couple to, and decay into, magnetosonic modes in the field reversal and in the wake of the plasmoid where the (weak) magnetic field is directed along $z$. This process leads to evolved MHD plasma turbulence in the sense of Tsytovich (1977). In our simulation there is no dissipation scale because particle collisions are absent, so that the power law extends to the smallest resolved scales.

\section{Magnetic Time Series}

In the course of the simulation, the complete magnetic fields $\mathbf{B}(x, z)$ are stored every $\Omega_{i}^{-1}$ and magnetic time series at given positions are extracted. In real time, the Nyquist frequency is about $0.75 \mathrm{~Hz}$. Since the most characteristic features arise at $t>200 \Omega_{i}^{-1}$ when lobe plasma becomes reconnected, we consider this stage below. Also, data from $x \lesssim 50 \lambda_{0}$ are affected by numerical boundary conditions at $x=0$ and are therefore disregarded. 

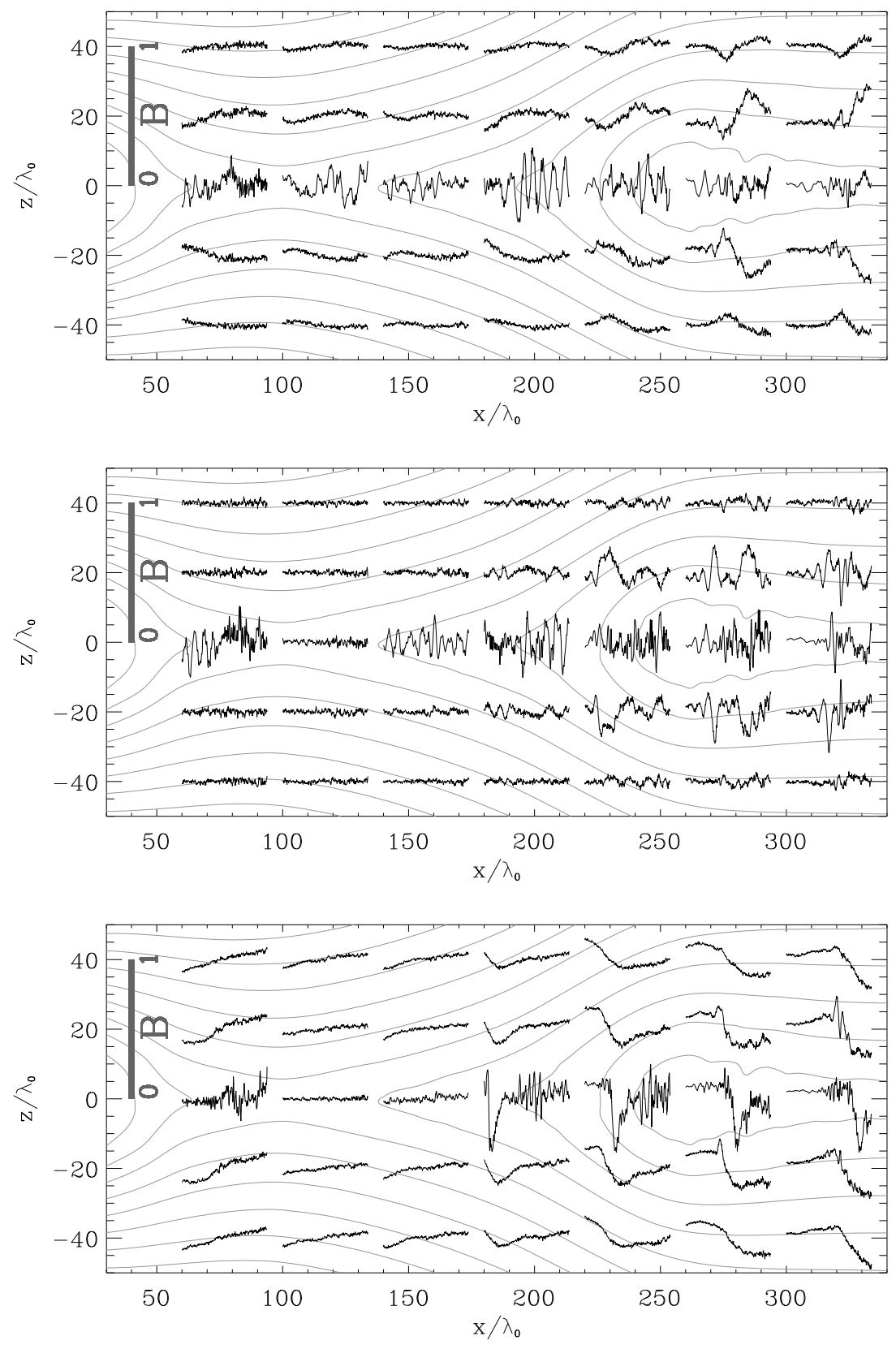

Fig. 4. Black curves: simulated magnetic time series $\left(240 \Omega_{i}^{-1} \leq t \leq 500 \Omega_{i}^{-1}\right)$ at different positions in the $x z$-plane. Gray: projection of the magnetic field lines onto the $x z$-plane at $t=360 \Omega_{i}^{-1}$. See text.

Figure 4 shows typical examples of time series of $B_{x}$ (top), $B_{y}$ (middle) and $B_{z}$ (bottom) recorded at different positions in the $x z$-plane. The solid curves represent the time interval $240 \Omega_{i}^{-1} \leq t \leq 500 \Omega_{i}^{-1}$, ranging from 4 to $8.3 \mathrm{~min}$ utes after reconnection onset in real time. For comparison, the field lines at an intermediate time $t=360 \Omega_{i}^{-1}$ are also given (dotted). Each curve corresponds to the position of their left starting point and all curves have the same scale for the magnetic field (gray bar: lobe field strength). A closeup of representative time series is shown in Fig. 5, together with the in-plane bulk velocity field at $t=360 \Omega_{i}^{-1}$. The position of each time series (bottom) is marked by a star in the top panel.

From the study of an extended set of time series similar to Fig. 4, the following picture emerges. The magnetic field in the inflow region $\left(|z| \gtrsim 0.5\left|x-x_{\eta}\right|\right.$; Fig. 5 top $)$ is calm and carries only weak $(\leq 0.05)$ statistical fluctuations. The immediate vicinity of the $\mathrm{X}$ point with nonvanishing resistivity (Fig. 5, ' $\mathrm{X}$ ') is characterized by strong fluctuations in $B_{x}$ on a time scale of $20 \Omega_{i}^{-1}$, whereas $B_{y}$ and $B_{z}$ are only weakly noisy with amplitude $\lesssim 0.05$. In the downtail equatorial plane at $x-x_{\eta} \gtrsim 75$ (Fig. 5, 'A'), a plasmoid has evolved and is ejected tailwards; its passage manifests in a distinct peak in $B_{z}$ and in quasi-coherent oscillations in $B_{y}$ (not shown) and $B_{x}$ (shown in Fig. 5) in the post-plasmoid plasma sheet. Contrary to the classical bipolar plasmoid signature in $B_{z}$, the present signature has a unipolar structure because there is no second distant X point, so that the observer is passed by the lefthand plasmoid boundary only. Outside the equatorial plane, the plasmoid signature in $B_{z}$ becomes smoother 

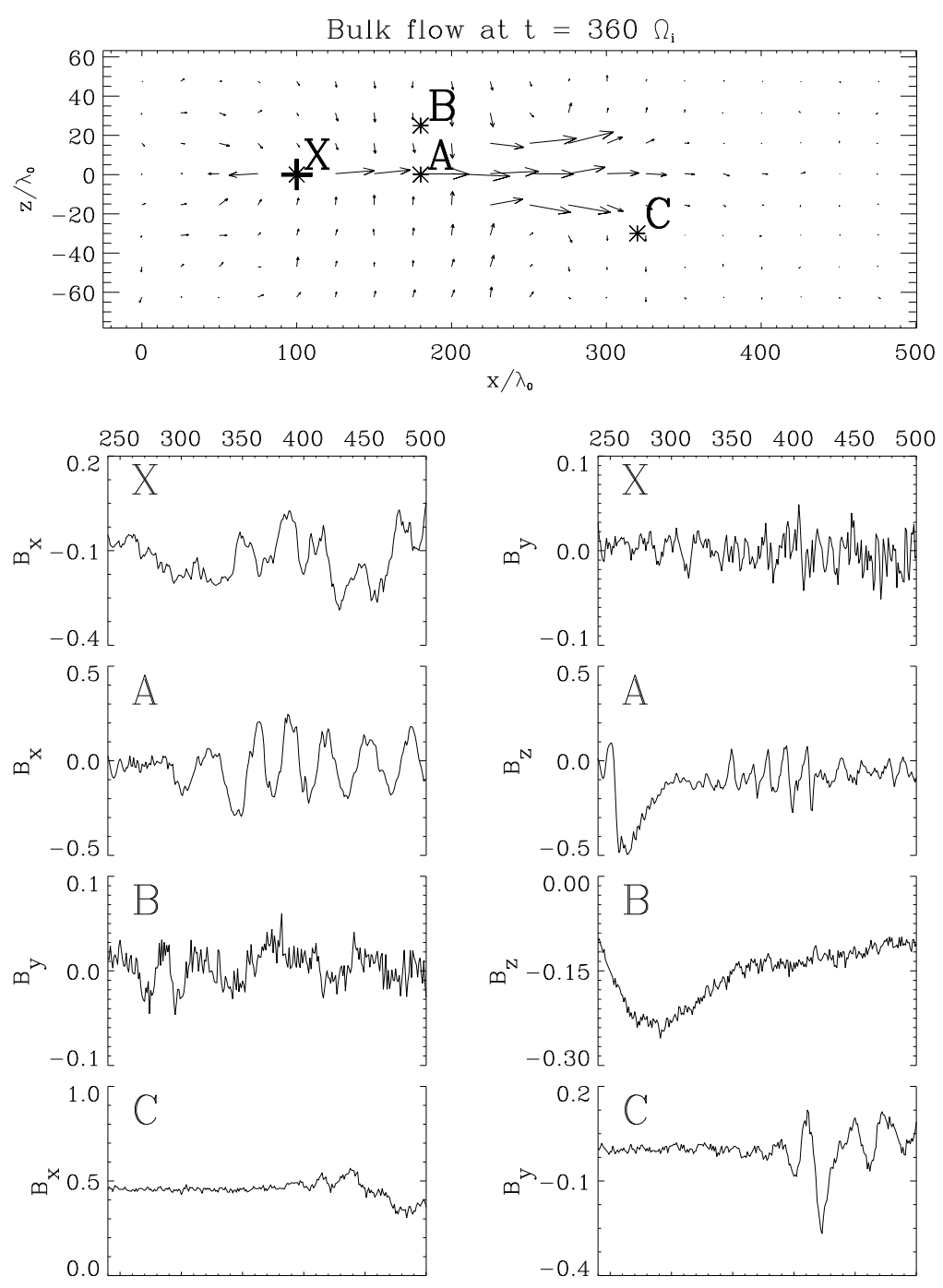

Fig. 5. Top: In-plane velocity field at $t=360 \Omega_{i}^{-1}$ and positions (star) of simulated magnetic time series X,A,B,C (bottom).

with increasing $|z|$, whereas $B_{y}$ remains fluctuating (Fig. 5, ' $\mathrm{B}$ '). With increasing $x$, the quasi-periodic oscillations in $B_{x}$ vanish and the plasmoid passage is most distinctly seen in $B_{y}$ (Fig. 5, 'C').

Since the plasmoid moves into the $+x$-direction, its passage appears delayed at subsequent $x$. The resulting signature in the center $(z=0)$ and lobe $(z=20)$ are shown in Fig. 6. All curves have the same scale, and different curves refer to different $x$-positions (middle labels). In the center of the plasma sheet, the dominant feature is a negative peak in $B_{z}$ due to piled-up field lines in the wake of the plasmoid, whereas $B_{y}$ and $B_{x}$ show merely an increase in the fluctuations when the observer is passed by the post-plasmoid current sheet. The lobe signatures are much smoother, and the largest modulations occur in the Hall field $B_{y}$.

In order to characterize the turbulent pattern in the PPPS time series, their temporal power spectra were computed using a Hanning window (Fig. 7). The spectra represent spatial averages over the regions $150 \lambda_{0} \leq x \leq 300 \lambda,|z| \leq 5 \lambda_{0}$ in logarithmic scale. They show moderate peaks at $\omega \sim 0.03$ and 0.07 , which correspond to the distinct wavelengths in Fig. 3 bottom middle, advected with the bulk velocity (since the shape of the spatial structures evolves in time, they do not transform to equally distinct peaks in the temporal spectra). The spectra can be represented by two power laws (boldface dashed lines), separated by a turnover frequency $\omega^{*} \approx 0.02 \Omega_{i} \sim 0.03 \mathrm{~Hz}$. At $f<f^{*}$, the power law index is about 1.3 and at $f>f^{*}$, the power law index is 2.2. Whereas power law behaviour at $f<f^{*}$ can be questioned on grounds of the limited data points and windowing effects, the spectra follow at $f>f^{*}$ a power law with remarkable accuracy. We interpret this as the signature of evolved or almost evolved MHD turbulence (Subsection 3.1), starting from the input scale prescribed by the linear instability of the PPPS. The simulated power law index $(\alpha \sim 2.2)$ is in qualitative agreement with the theoretical estimate $(\alpha \sim 2)$ given by Tsytovich (1977) for the steady-state spectrum of fast magnetosonic turbulence.

The power law behaviour found in our simulation is compatible with the results of Hoshino et al. (1994), who found from GEOTAIL observations in the distant magnetotail a turnover frequency $f^{*}$ in the range of $0.02 \mathrm{~Hz}$ to $0.08 \mathrm{~Hz}$, with power law slopes of 1.2 to 1.8 below, and 1.9 to 3.3 above $f^{*}$, respectively. It also agrees with the temporal 

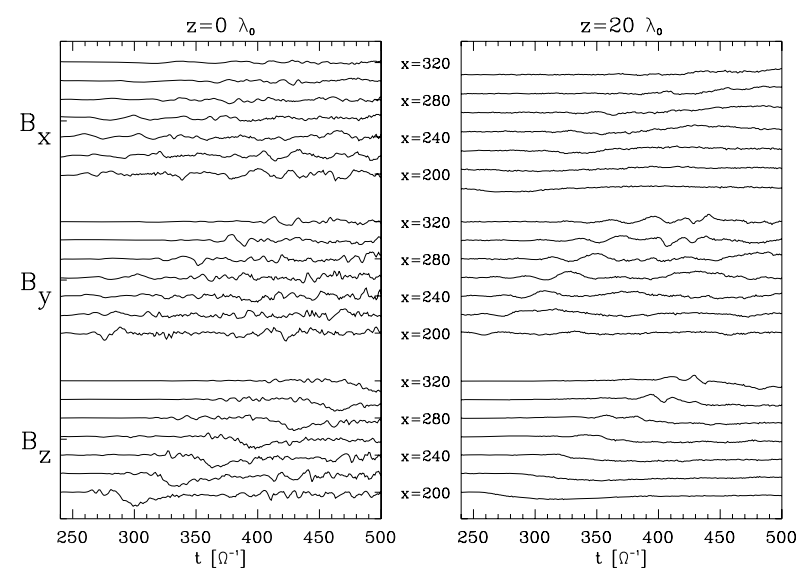

Fig. 6. The simulated passage signature of the plasmoid at different positions in space.

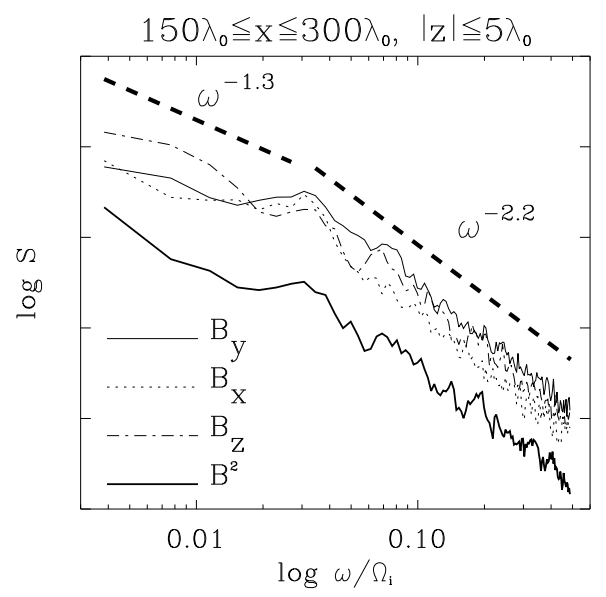

Fig. 7. The simulated power spectra of $B_{x}, B_{y}, B_{z}$ and $B^{2}$ time series, averaged over the turbulent range of the post-plasmoid plasma sheet. In real time, $\Omega_{i}$ corresponds to about $1.5 \mathrm{~Hz}$.

power spectra of near-Earth AMPTE observations (Bauer $e t$ al., 1995), yielding a turnover frequency of $f \sim 0.03 \mathrm{~Hz}$ with power law slopes of 1.5 below and 2 to 2.5 above $f^{*}$, although the latter observations were made on the Earthward side of, and thus closer to, the reconnection point.

\section{Summary and Conclusions}

Hybrid simulations of magnetotail reconnection have resulted in highly anisotropic particle distributions within the reconnection layer. The kinetic cause of the velocity anisotropy is the simultaneous presence of inflowing lobe ions and outflowing ions deflected in the field reversal. The resulting velocity $\left(T_{\|}>T_{\perp}\right)$ anisotropy drives a firehose type instability exciting mostly Alfvénic perturbations, which couple to, and decay, into magnetosonic modes due to nonlinear interaction and the inhomogeneity of the system. This process finally leads to a filamentation and disruption of the PPPS and to evolved or almost evolved MHD turbulence in the wake of the plasmoid. The linear stage of the instability can be described by an anisotropic 3-layer fluid model where the free energy is provided by the velocity anisotropy in the PPPS and the wave vector of maximum growth is determined by the model geometry.

In the present paper we have analyzed magnetic time series obtained from these hybrid simulations which can be compared with spacecraft observations. The diffusion region manifests itself in strong fluctuations in $B_{x}$, whereas $B_{y}$ and $B_{z}$ remain calm; the downtail equatorial region shows a (unipolar) plasmoid signature in $B_{z}$, followed by quasicoherent oscillations in $B_{x}$ and $B_{y}$ with period $\sim 1$ minute, which become turbulent at $x-x_{\eta} \gtrsim 150 \lambda_{0}$. The plasma sheet boundary layer carries fluctuations in the Hall field $B_{y}$, whereas $B_{x}$ and $B_{z}$ show smooth signatures of plasmoid passage. In the lobe, all signatures become smooth and the largest modulations occur in $B_{y}$. It is found that temporal power spectra of the PPPS turbulence at fixed position agree remarkably well with GEOTAIL (Hoshino et al., 1994) and AMPTE (Bauer et al., 1995) satellite observations.

The analysis of the full simulated space-time data reveals that the quasi-coherent oscillations in PPPS time series are due to the linear stage of the PPPS instability, which is characterized by quasi-monochromatic waves traveling tailwards with about the outflow velocity. This offers the possibility that the quasi-coherent oscillations seen in spacecraft observations are not the signature of a sequence of plasmoids (advected tearing modes), as might be suspected from the time series of an isolated probe in the equatorial plane (Hoshino, 2000), but are due to MHD waves excited by the mechanism described above.

We should finally stress that our simulation is limited with regard to its two-dimensionality and the neglection of electron dynamics. It is to be expected that three-dimensional simulations including electron dynamics will reveal the lower hybrid drift instability, which is not resolved within the present simulation. Also, there are alternative explanations of turbulent appearance of the spacecraft time series, such as flapping motions of the whole magnetotail. Such alternative scenarios are not ruled out by the present simulation.

Acknowledgments. We thank Rudolf A. Treumann for helpful comments and discussions.

\section{References}

Angelopoulos, V., W. Baumjohann, C. F. Kennel, F. V. Coroniti, M. G. Kivelson, R. Pellat, R. J. Walker, H. Lühr, and G. Paschmann, Bursty bulk flows in the inner central plasma sheet, J. Geophys. Res., 97, 40274039, 1992.

Arzner, K. and M. Scholer, Kinetic structure of the post-plasmoid plasma sheet during magnetotail reconnection, J. Geophys. Res., 106, 38273844, 2001.

Bauer, T., W. Baumjohann, R. Treumann, N. Sckopke, and H. Lühr, Lowfrequency waves in the near-Earth plasma sheet, J. Geophys. Res., 100, 9605-9617, 1995.

Birn, J., R. Sommer, and K. Schindler, Open and closed magnetospheric tail configurations and their stability, Astrophys. and Space Science, 35, 389-402, 1975.

Borovsky, J. E., R. C. Elphic, H. O. Funsten, and M. F. Thomsen, The Earth's plasma sheet as a laboratory for flow turbulence in high- $\beta$ MHD, J. Plasma Physics, 57, 1-34, 1997.

Gary, P., Theory of space plasma microinstabilities, 181 pp., Cambridge Univ. Press, 1993.

Hoshino, M., A. Nishida, T. Yamamoto, and S. Kokobun, Turbulent magnetic field in the distant magnetotail: bottom-up process of plasmoid formation?, Geophys. Res. Lett., 21, 2935-2938, 1994.

Hoshino, M., Small scale plasmoids in the post-plasmoid plasma sheet: 
origin of MHD turbulence?, Adv. Space Res., 25, 1685-1688, 2000.

Karimabadi, H., D. Krauss-Varban, and N. Omidi, Magnetic structure of the reconnection layer and core field configuration in plasmoids, J. Geophys. Res., 104, 12,3131-12,326, 1999.

Krauss-Varban, D. and N. Omidi, Large-scale hybrid simulations of the magnetotail during reconnection, Geophys. Res. Lett., 22, 3271-3274, 1995.

Kropotkin, A. P., O. O. Trubachev, and A. T. Y. Lui, Nonlinear instability of the geomagnetic tail current sheet combining the features of tearing and cross-field current instabilities, J. Geophys. Res., 104, 371-381, 1999.

Kuznetsova, M., M. Hesse, and D. Winske, Ion dynamics in a hybrid simulation of magnetotail reconnection, J. Geophys. Res., 101, 27,351$27,373,1996$.

Lin, Y. and D. W. Swift, A two-dimensional hybrid simulation of the magnetotail reconnection layer, J. Geophys. Res., 101, 19,859-19,870, 1996. Lottermoser, R.-F. and M. Scholer, Hybrid simulation of magnetotail reconnection, Phys. Chem. Earth (C), 24, 275-279, 1999.
Matthews, A. P., Current advance method and cyclic leapfrog for 2D multispecies hybrid plasma simulations, J. Comp. Phys., 112, 102-116, 1994

Milovanov, A. V., L. M. Zelenyi, and G. Zimbardo, Fractal structures and power law spectra in the distant Earth's magnetotail, J. Geophys. Res., 101, 19,903-19,910, 1996.

Sundaram, A. K. and D. H. Fairfield, Localized tearing modes in the magnetotail driven by curvature effects, J. Geophys. Res., 100, 3563-3572, 1995

Tsytovich, V. N., Theory of turbulent plasma, 535 pp., Consultants Bureau, New York, 1977.

Zwingmann, W., J. Wallace, K. Schindler, and J. Birn, Particle simulation of magnetic reconnection in the magnetotail configuration, J. Geophys. Res., 95, 20,877-20,888, 1990.

K. Arzner (e-mail: kja@mpe.mpg.de) and M. Scholer 